\title{
Conservative surgery of the mitral valve in bacterial endocarditis
}

\author{
MICHAEL D GAMMAGE, WILLIAM A LITTLER, LEON D ABRAMS \\ From the University Department of Cardiovascular Medicine and East Birmingham Hospital, Birmingham
}

ABSTRACT Surgical repair of the mitral valve was undertaken in two young female patients during the active phase of bacterial endocarditis to eradicate persistent infection. Operation resulted in rapid resolution of infection with good haemodynamic results in both patients. A mitral valve prosthesis has the disadvantage for children of needing replacement because of growth, and for young women of leading to problems in pregnancy because of the need for lifelong anticoagulant treatment. Thus repair rather than replacement of the mitral valve should be considered in patients, especially young women, presenting with mitral regurgitation in these circumstances.

Early surgical intervention in bacterial endocarditis is well established. ${ }^{2}$ In most cases the infected or damaged valve is excised and replaced with a prosthesis.

Prosthetic valve replacement in the younger patient, however, presents several problems of long term management. The life expectancy of prosthetic valves remains unknown and this uncertainty, combined with the need to replace prosthetic valves in children who have outgrown an initial prosthesis, presents the daunting prospect of further operation. The lifelong anticoagulant treatment required by patients with prosthetic valves is not without risk and in young women the likelihood of pregnancy adds further complications.

In younger patients therefore repair of a damaged valve may be more appropriate in selected cases. We report two cases in which surgical repair of the affected valve was performed with good results.

\section{Case reports}

CASE 1

A 10 year old caucasian girl presented with a one week history of sore throat, pyrexia, and general malaise. She was previously fit with no important medical history. On examination her temperature was $39.5^{\circ} \mathrm{C}$ and she was drowsy and dehydrated.

Address for reprint requests: Dr MD Gammage, Department of Cardiovascular Medicine, East Birmingham Hospital, Birmingham B9 5ST.

Accepted 25 May 1984
Her pulse rate was 140 beats/min (sinus rhythm) and her blood pressure $100 / 60 \mathrm{mmHg}$, with no signs of cardiac failure. Auscultation revealed a loud, pansystolic murmur at the apex and left sternal edge. A blood film showed a neutrophil leucocytosis with a left shift. Blood cultures subsequently grew Staphylococcus aureus. Despite appropriate antibiotic treatment her condition deteriorated. She developed multiple petechial skin lesions, spasticity of the lower limbs, and progressive cardiac failure. Computed tomography showed appreciable cerebral oedema but no focal lesions. Two dimensional echocardiography showed a large vegetation $(1 \mathrm{~cm}$ in diameter) on the anterior leaflet of the mitral valve (fig 1). There was evidence of mitral valve prolapse and a small pericardial effusion.

Despite aggressive treatment with digoxin, diuretics, and high dose intravenous antibiotics (flucloxacillin $200 \mathrm{mg}$ four hourly, fusidic acid $500 \mathrm{mg}$ daily) her pyrexia did not settle; cardiac failure persisted and tricuspid incompetence developed. Persistent diarrhoea, suggestive of pseudomembranous colitis, required a change in antibiotic treatment and vancomycin was substituted, although Clostridium difficile was not isolated. After about a month of conservative treatment with little improvement, she was referred for a surgical opinion and an open mitral valve repair was performed.

Median sternotomy and pericardotomy were performed, the surface of the heart appearing inflamed and showing telangiectases. The left ventricle was vented and the left atrium opened. There was a large, solid, smooth mass of thrombus ("vegetation") about $2 \times 1 \times 1 \mathrm{~cm}$ on the upper surface and 

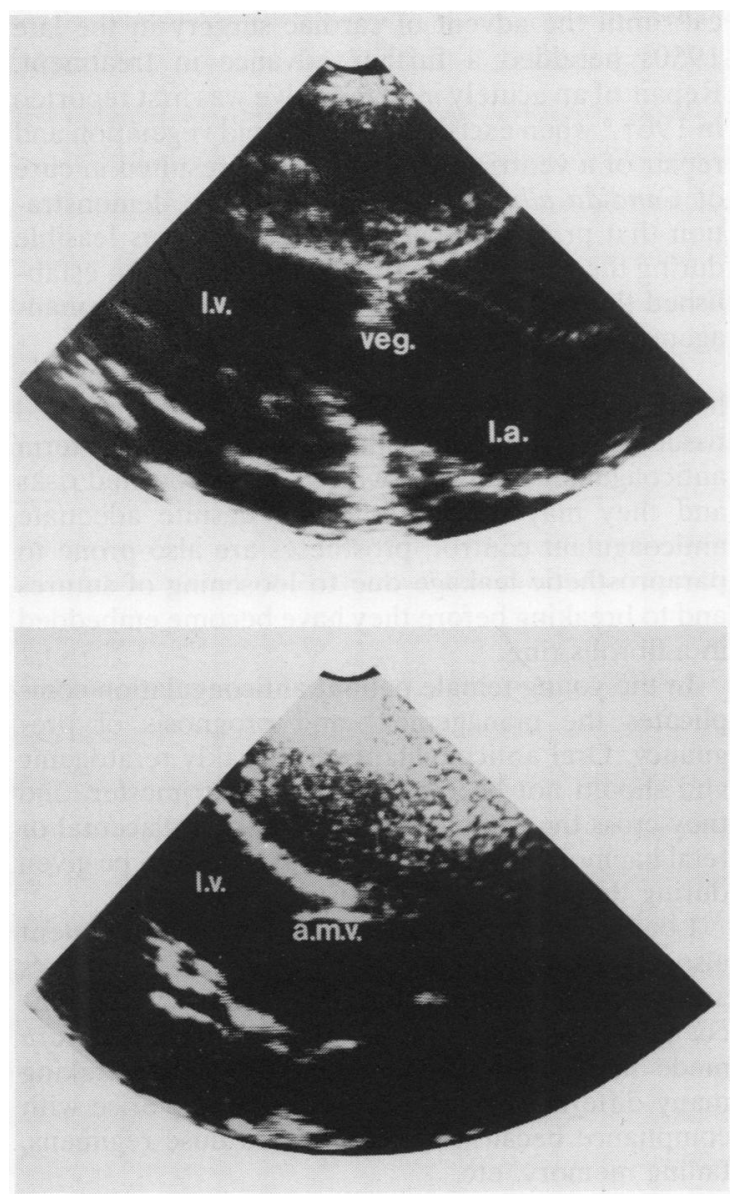

Fig 1 Two dimensional echocardiogram (parasternal view, long axis) showing (a) vegetation on anterior mitral valve leaflet, with a small pericardial effusion; (b) postoperative appearance showing some thickening of anterior mitral valve leaflet but no vegetation. l.v.-left ventricle; l.a.-left atrium; veg.-vegetation;

a.m.v. - anterior mitral valve leaflet.

edge of the lateral commissure. This was excised and some rather firmer vegetation was scraped off the posterior cusp; this left the valve with a slight deficiency of the lateral commissure, which appeared to be due to longstanding, localised thickening and shrinking of that part of the mitral valve. This deficiency was repaired by a localised annuloplasty at the lateral commissure, a heavy mattress suture being used to shorten the mitral annulus. The atrial wall was folded over the repair to reinforce it. The valve was then tested by injecting fluid through the left ventricular vent. The cusps themselves were largely spared from inflammation. The patient sub- sequently made a good recovery and was well at follow up. Gram stain of the vegetations showed Gram positive cocci consistent with a staphylococcus. Histological examination of the tissue excised with the vegetation showed thickening by granulation tissue with a superimposed fibrinous exudate. The granulation tissue showed capillary proliferation and infiltration by fibroblasts and lymphocytes. The exudate included neutrophils and mononuclear cells, but no organisms.

\section{CASE 2}

A 20 year old caucasian woman presented with a one week history of ankle swelling, pleuritic chest pain, and persistent tachycardia after an influenza like illness. She had had scarlet fever as a child. On examination she was febrile and had a single splinter haemorrhage on the right thumb. Her pulse rate was 130 beats/min with sinus tachycardia and blood pressure $110 / 70 \mathrm{mmHg}$. She had clinical cardiomegaly and auscultation revealed a loud pansystolic murmur at the apex, radiating to the whole precordium. The lungs were clear. A blood film showed a hypochromic anaemia (haemoglobin concentration $9.4 \mathrm{~g} / \mathrm{dl})$ and a neutrophil leucocytosis $\left(13.6 \times 10^{9} /\right.$ 1). The erythrocyte sedimentation rate was raised at $85 \mathrm{~mm}$ in one hour (Westergren). Blood culture grew an $\alpha$ haemolytic streptococcus. She was treated with intravenous benzylpenicillin. Echocardiography showed mitral valve prolapse with dense echoes on the posterior leaflet in systole, suggestive of vegetations (fig 2). Gentamicin was subsequently added to her regimen, but after three weeks there had been little improvement, with continuing pleuritic chest pain, tachycardia, and pyrexia. Her antibiotic treatment was changed to erythromycin with initial improvement, but her pyrexia recurred and she had an embolic episode in the left leg. Pyrexia persisted and surgery was undertaken.

Median sternotomy and pericardiotomy were performed and the left ventricle was found to be enlarged. There was a tear in the medial part of the anterior cusp of the mitral valve, with one adjacent chord ruptured and some ballooning of the cusp adjacent to the tear. There was some adherent thrombus on the damaged part of the anterior cusp. The posterior cusp showed only a little thickening and a minor degree of shortening of the chordae adjacent to the medial commissure. The localised ballooned part of the mitral valve was excised and the tear repaired by suturing it to the appropriate part of the papillary muscle; the deficiency in the medial commissure caused by the lesion in the posterior cusp was repaired by shortening the annulus with a heavy mattress suture at the medial commissure. 

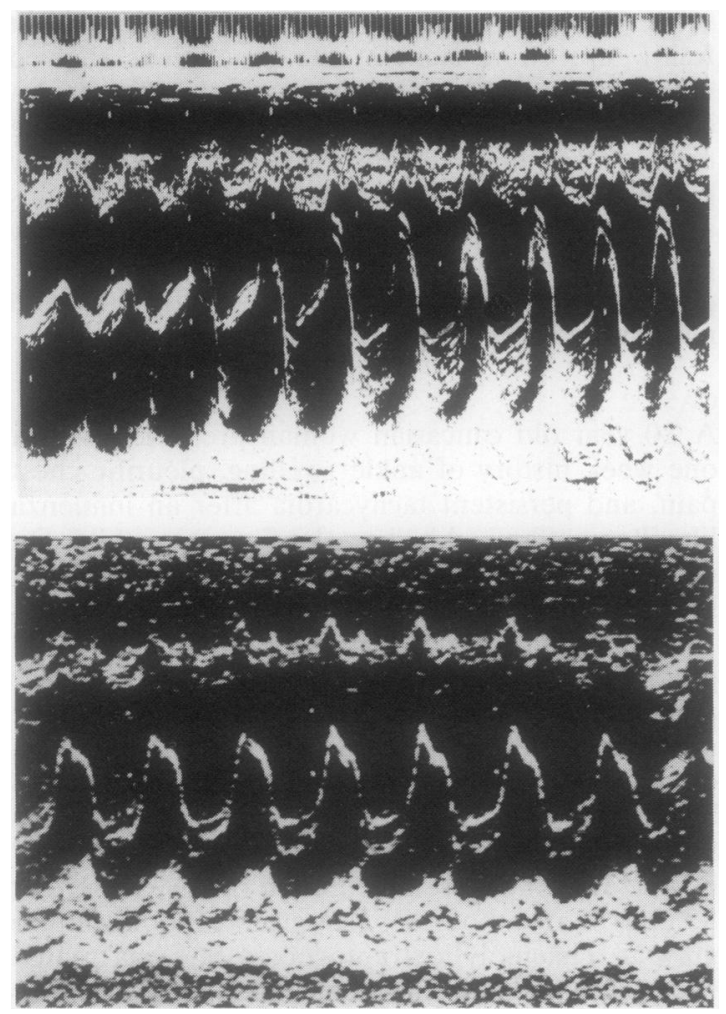

Fig $2 M$ mode echocardiogram showing (a) dense echoes associated with the posterior mitral valve leaflet, suggestive of vegetation, with mitral valve prolapse also present; (b) mitral valve prolapse persisting after operation, but the posterior leaflet now appearing normal.

The patient subsequently made a good recovery and was well apart from absent left foot pulses at follow up. Culture of the excised valve thrombus grew an $\alpha$ haemolytic streptococcus and histological examination of the excised valve tissue showed cellular fibrosis with scattered acute and chronic inflammatory cells. There was considerable vascularisation of the valve and superimposed thrombotic material, within which Gram positive cocci were seen.

\section{Discussion}

It is nearly 100 years since William Osler discussed valvular endocarditis in a series of Goulstonian lectures $^{3}$ and over 40 years since the first surgical repair of a patent ductus arteriosus resulting in a cure of bacterial endocarditis without further treatment. ${ }^{4}$ With the arrival of the antibiotic era, the treatment of infective endocarditis was predominantly medi- $\mathrm{cal}^{5}$ until the advent of cardiac surgery in the late 1950s heralded a further advance in treatment. Repair of an acutely infected valve was first reported in $1961,{ }^{6}$ when excision of a tricuspid vegetation and repair of a ventricular septal defect resulted in cure of Candida albicans endocarditis. The demonstration that prosthetic valve replacement was feasible during the active phase of infection, however, established the importance of this procedure in the management of infective endocarditis.

Although providing rapid improvement in haemodynamics and allowing excision of all infected tissue, mechanical prostheses require long term anticoagulant treatment with all the associated risks and they may develop thrombi despite adequate anticoagulant control; prostheses are also prone to paraprosthetic leakage due to loosening of sutures and to breaking before they have become embedded in a fibrous ring.

In the young female patient anticoagulation complicates the management and prognosis of pregnancy. Oral anticoagulants are weakly teratogenic and should not be given in the first trimester, and they cross the placenta with the risk of placental or fetal haemorrhage and should not therefore be given during the last few weeks of pregnancy.

Long term anticoagulation in the older patient also presents problems, as many of these patients are having medication that may interfere with anticoagulant control, particularly when changes are made in dosage. These patients are often taking many different drugs and problems may arise with compliance because of complicated dose regimens, failing memory, etc.

A further problem in the young patient is that of mechanical failure of the prosthesis due to wear and in the very young the prosthesis may need replacement owing to growth of the heart. Heterograft prostheses avoid some of these problems, but remain something of an unknown quantity with regard to life expectancy.

Both our patients had echocardiographic evidence of mitral valve prolapse, which we assume to be the predisposing lesion, and they presented with clinical evidence of mitral regurgitation and subsequent pulmonary oedema. Both had long periods of "aggressive" medical treatment before surgical intervention, which was performed primarily to remove the persisting focus of infection.

Repair of the mitral valve is more frequently possible than aortic valve repair; ${ }^{1}$ this is because infection of the mitral valve can be more easily eliminated and mitral valvular ring abscesses are much less common. ${ }^{27}$

Manhas $e^{a l^{8}}$ in 1971 reviewed the early and late results in 91 patients undergoing reconstructive 
surgery for mitral incompetence, although the aetiology was not stated. They concluded that this was the treatment of choice for patients with ruptured chordae and pure mitral regurgitation and that prosthetic valve replacement was indicated for a badly scarred, calcified, or stenotic valve, or if the annulus was thinned or weakened. They also pointed out that a degree of residual regurgitation is common and requires differentiation from recurrent regurgitation.

Adebo and Ross ${ }^{9}$ in 1983 reviewed their experience over five years with mitral valve reconstruction by the Carpentier method, ${ }^{10}$ in which the mitral annulus is remodelled on a prosthetic ring. They confirmed that acceptable functional improvement was obtained and that the method was applicable regardless of aetiology. The valve failure rate was less than $7 \%$ at 3.5 years and anticoagulation was indicated only if the patient was in atrial fibrillation.

In conclusion, we suggest that repair of the mitral valve should be considered in patients presenting with mitral regurgitation as a result of infective endocarditis who require operation during the acute phase because of uncontrolled infectionparticularly young women, in whom the avoidance of anticoagulant treatment will improve the prognosis of any subsequent pregnancy.

We wish to thank Miss AM Strong for her help in typing the manuscript.

\section{References}

' Parrot JCW, Hill JD, Kerth WJ, Gerbode F. The surgical management of bacterial endocarditis. Ann Surg 1976; 183:289-92.

${ }^{2}$ Mills SA. Surgical management of infective endocarditis. Ann Surg 1982;195:367-83.

${ }^{3}$ Osler W. Goulstonian lectures on malignant endocarditis. Lancet 1885; i:415-8, 459-64, 505-8.

${ }^{4}$ Touroff ASW, Vessell H. Sub-acute Strep viridans endarteritis complicating patent ductus. arteriosus. Recovery following surgical treatment. JAMA 1940; 115: 1270-2.

${ }^{5}$ Loewe L, Rosenblatt P, Greene HJ, Russell M. Combined penicillin and heparin therapy of sub-acute bacterial endocarditis. Report of seven consecutive successfully treated patients. JAMA 1944;124:144-9.

${ }^{6}$ Kay JH, Bernstein S, Feinstein D, Biddle M. Surgical cure of Candida albicans endocarditis with open-heart surgery. $N$ Engl J Med 1961;264:907-10.

${ }^{7}$ Arnett EN, Roberts WC. Valve ring abscess in active infective endocarditis. Frequency, location and clues to clinical diagnosis from the study of 95 necropsy patients. Circulation 1976;54:140-5.

${ }^{8}$ Manhas DR, Rittenhouse EA, Hessell EA, Merendius KA. Reconstructive surgery for the treatment of mitral incompetence. Early and late results in 91 patients. J Thorac Cardiovasc Surg 1971;62:781-7.

9 Adebo OA, Ross JK. Conservative surgery for mitral valve disease: clinical and echocardiographic analysis of the results. Thorax 1983;38:565-71.

${ }^{10}$ Carpentier A, Deloche A, Dauphin J, et al. A new reconstructive operation for correction of mitral and tricuspid valve insufficiency.J Thorac Cardiovasc Surg 1971;61:1-13. 